\title{
Nephrophobia: teaching renal medicine to the undergraduate student
}

\author{
Siôn Edryd Williams $s^{1,2}$
}

Received: 21 August 2018 / Accepted: 22 August 2018 / Published online: 28 August 2018

(c) Italian Society of Nephrology 2018

As a second year medical student, I can recall a palpable lack of enthusiasm for a half-day symposium on the 'Basics of Renal Physiology and Histology: Part 1'. The lecture theatre was barely half-full, the British skies were very grey, and we were about to sit quietly for our final teaching of term before the winter holiday. The afternoon unfolded with an introductory lesson to the histology of the renal glomerulus (supplemented by a number of pink-and-purple stained brightfield micrographs to wet the appetite, of course), a particularly dull session on ionic tubular transport, and then a quiz (hoorah) on the regulation of plasma and volume osmolarity. This was the first of a series of three symposia, the third of which I could be certain I'd be the only one attending.

As I immerse myself into higher postgraduate training in renal medicine, and as an advocate of good medical education, I have subjected myself to some heavy reflection on the quality of nephrology undergraduate education in both the pre-clinical (the 'basic sciences'-type) and clinical settings. Infrequent do I come across an undergrad excited by the renal sciences or clinical nephrology. In fact, it is often condemned (along with biomedical statistics, virology, the complement cascade and molecular-level oxygen homeostasis...remember Krebs?) to the very bottom of any preexam revision file. I suspect most student feel frankly overwhelmed by the intricacies of renal physiology, hormonal cascades, the interpretation of electrolyte imbalances and any brief mention of genetic syndromes with a renal flavour (see Liddle's, Gitelmann's, and Barrter's), and of course, there is absolutely no doubt that they are difficult subjects to master; it takes years of higher specialty training to competently and confidently apply theory and knowledge to manage clinical encounters. Nonetheless undergraduate training seems to be a key period to harbour a more positive and

Siôn Edryd Williams

sion.williams@gtc.ox.ac.uk

1 Oxford University Hospitals NHS Foundation Trust, Oxford, UK

2 Green Templeton College, University of Oxford, Oxford, UK empowering experience of medical specialties; the lack of exposure and the often too complex (and frankly, dull) ways in which kidney knowledge is disseminated to the masses solidifies a negativity towards the subject (which is unfortunately then carried through to future doctoring careers) [1]. Indeed, according to a survey of interns in the States, poor teaching is stipulated to be a significant contributor to the current shortfall in applications to nephrology [2]. So, what should we do?

Medical education has developed tremendously. Advances in both the science of learning and teaching practices has opened the door to more engaging and effective nephrology education [3]. My hope for the future is that undergraduate leaders can implement teaching strategies and modalities that are much more attractive to doctors in training, stimulate a curiosity rather than fear in the subject, and empower their students with knowledge that they then retain and can apply in practice.

Didactic, dull, one-way teaching is old, and interactivity is paramount if we are to prove that nephrology education is alive and thrilling for our undergraduates. Previous studies in renal education have suggested an appreciation amongst students for small-group exercises [4], 'clicker' poll quizzes for larger groups [5], and in junior grade renal doctors there has been great success with platforms which allow healthyheated debates to stimulate the academic discourse [6]. Utilised carefully to avoid overwhelming junior students, the use of clinical preambles to introduce first-principle renal science has been successful $[5,7,8]$. There has also been success in telemedical teaching of kidney ethics, where students are Skype-linked to a patient to discuss quality of life and experiences on dialysis [9].

The use and availability of free, open-access medical education resources is increasing. Social media is at the forefront of nephrology education albeit primarily for postgraduates. International societies (including the American Society of Nephrology, National Kidney Foundation and the European Renal Association-European Dialysis and Transplantation Association) have adopted various platforms to deliver its materials and teach; from online microblogging, games, 
visual abstracts, mentorships, and live tweeting for participative learning-it is not completely outlandish to suggest that this style of teaching could be applied and utilised in the undergraduate setting. There is of course undoubted heterogeneity in the way in which medical students engage with learning; would a nephrology curriculum that engages with the breadth of learning styles be beneficial? Internet-based social-media-type teaching provides flexibility and allows for more immersive learning, an opportunity to consolidate outside the usual classroom or clinical environment.

Lets make undergraduate kidney teaching great. Lets enthuse, engage and empower our students by being interactive and flexible with our teaching styles. Lets promote immersive learning by increasing their exposure to the clinical specialty (or at least once in their early training). Lets not teach for hours on the intricacies of Liddle's, Gitlemann's and Barrter's.

Nephrology is an incredible specialty; taught well, students should see that too.

\section{Compliance with ethical standards}

Conflict of interest The author declares he has no conflict of interest.

Ethical approval This article does not contain any studies with human participants performed by any of the authors.

Informed consent For this type of study, formal consent is not required.

\section{References}

1. Patel AB, Balzer MS (2013) On becoming a nephrologist: medical students' ideas to enhance interest in a career in nephrology. Am J Kidney Dis [Internet] 62(3):450-452. https://doi.org/10.1053/j. ajkd.2013.01.020
2. Jhaveri KD, Sparks MA, Shah HH, Khan S, Chawla A, Desai T et al (2013) Why not nephrology? A survey of US internal medicine subspecialty fellows. Am J Kidney Dis [Internet] 61(4):540 546. https://www.ncbi.nlm.nih.gov/pmc/articles/PMC4164433/ pdf/nihms626778.pdf. Accessed 31 July 2018

3. Helbert RB, Johnston JR (2016) Applying effective teaching and learning techniques to nephrology education [Internet]. Clin Kidney J 9:755-762. https://watermark.silverchair.com/sfw08 3.pdf?token=AQECAHi208BE49Ooan9kkhW_Ercy7Dm3ZL _9Cf3qfKAc485ysgAAAZswggGXBgkqhkiG9w0BBwagggGI MIIBhAIBADCCAX0 GCSqGSIb3DQEHATAeBglghkgB ZQMEAS4wEQQMGuwBn0kFDPCFm38JAgEQgIIBTgFQ D5pD8Vg1JRLuS0RHInBiOf3zq1HPfZL6PmuKMfJfS56m. Accessed 31 July 2018

4. Brands MW, Schumacher L (2009) Active learning strategies to teach renal-cardiovascular integration with high student-to-teacher ratios. Adv Physiol Educ [Internet] 33(4):282-285. http://www. ncbi.nlm.nih.gov/pubmed/19948675. Accessed 31 July 2018

5. Dietz JR, Stevenson FT (2011) Active learning in a large medical classroom setting for teaching renal physiology. Adv Physiol Educ [Internet] 35(4):456-459. http://www.ncbi.nlm.nih.gov/pubme d/22139786. Accessed 31 July 2018

6. Jhaveri KD, Chawla A, Shah HH (2012) Case-based debates: an innovative teaching tool in nephrology education. Ren Fail [Internet] 34(8):1043-1045. http://www.ncbi.nlm.nih.gov/pubme d/22746167. Accessed 31 July 2018

7. Elzubeir MA (2012) Teaching of the renal system in an integrated, problem-based curriculum. Saudi J Kidney Dis Transpl [Internet] 23(1):93-98. http://www.ncbi.nlm.nih.gov/pubmed/22237226. Accessed 31 July 2018 (Medknow Publications and Media Pvt. Ltd.)

8. Harrison-Bernard LM (2009) The renal renin-angiotensin system. Adv Physiol Educ [Internet] 33(4):270-274. http://www.ncbi.nlm. nih.gov/pubmed/19948673. Accessed 31 July 2018

9. Bramstedt KA, Prang M, Dave S, Shin PNH, Savy A, Fatica RA (2014) Telemedicine as an ethics teaching tool for medical students within the nephrology curriculum. Prog Transpl [Internet] 24(3):294-297. http://journals.sagepub.com/doi/https://doi. org/10.7182/pit2014289 (SAGE Publications, Los Angeles) 